\title{
Causes and Countermeasures of Strong Vibration of Pipe End Thickener
}

\author{
Liu Tongyi \\ School of Mechanical Engineering, Shandong University of Technology, Zibo, China
}

\section{Email address:}

1ty-0611@163.com

\section{To cite this article:}

Liu Tongyi. Causes and Countermeasures of Strong Vibration of Pipe End Thickener. Industrial Engineering. Vol. 3, No. 2, 2019 , pp. $27-31$. doi: $10.11648 /$ j.ie.20190302.12

Received: April 4, 2019; Accepted: January 3, 2020; Published: January 13, 2020

\begin{abstract}
The pipe end thickening machine is a pressure processing equipment for thickening the pipe end of plastic material. The process of thickening the tubing end is to heat one end of the tubing to a certain temperature, push it into the die hall, and close the die cylinder under the push of hydraulic oil to press the end of the pipe. Then, squeeze into the cylinder forward, and the extrusion head on the piston rod thickens the end of the pipe. Complete the thickening process at the end of the pipe. In the production process, due to the great pressure change of the hydraulic system, the vibration of the system is violent, resulting in pipeline cracking, which seriously affects the production. Through the analysis of hydraulic system of pipe end thickener, it is found that the fundamental cause of the system pressure relief vibration is that when the hydraulic system is unloading, the volume of the hydraulic cylinder unloading cavity is large and the energy is accumulated. The problem can be solved by adjusting the section where the actual stroke of the hydraulic cylinder is located in the total stroke to reduce the volume of the pressure relief chamber and the energy released when the pressure is unloaded.
\end{abstract}

Keywords: Hydraulic System, Vibration Pressure, Release

\section{Overview}

Steel pipe end thickener is a kind of pressure processing equipment, which is used to thicken the pipe wall at one end of steel pipe. It is the main processing technology of oil pumping pipe manufacture in oil fields [1].

The process of pipe end thickening is to put one end of the pipe into the heating coil and then push it into the mold hall after heating to a certain temperature [2]. The closing cylinder begins to close the mold, pressing the end of the pipe [3]. Then, the cylinder starts to move, and the plunger on the piston rod thickens the end of the steel pipe. Complete pipe end thickening process.

During a working cycle, the system produces two severe vibrations, which seriously affects production as the vibration causes pipeline cracking.

\section{Hydraulic System Analysis}

(1) Starting motor

Press the motor start button, each electromagnet is not charged, each pump through the valve 2, 4, 6, 8, 10 unloading.
The motor starts without load.

(2) The main cylinder piston goes down quickly

Press the pressing button, the electromagnet YA2, YA3, YA4, YA5, YA6, YA10, YA11, YA13, YA14 gets the electricity, the five pumps all stop unloading, the discharged pressure oil passes through the one-way valve $1,3,5,7,9$, respectively, and then passes through the valve 19, 20 to enter the die closing cylinder cavity, and at the same time, the one-way valve above the die cylinder opens the liquid to the die closing cylinder, so that the piston rod of the die-closing cylinder drives the slider to fall quickly.

Slow down, close to the work piece

After the sliding block touches the quick-slow limiting switch, the electromagnet YA2, YA3, YA5, YA10 and YA15 are electrically connected, and the pressure oil pumped by the three plunger pumps enters the valve 19 through the one-way valves 1,3 and 7 , respectively, and then enters the upper cavity of the mold clamping cylinder, so that the piston rod of the mold clamping cylinder drives the sliding block to move slowly and downwards, and is close to the work piece.

(4) Closing, pressurizing

And the piston rod of the mold clamping cylinder drives the 
upper die holder and the lower die holder of the slide block to start clamping, and the workpiece is pressurized and molded. The pressing pressure at this time is regulated by the pressure relay $\mathrm{SP} 1$.

(5) Holding pressure

When the pressure of the die closing cylinder reaches the set value, the pressure relay sends out a signal. The electromagnets YA2, YA3, YA5, YA10 and YA15 are de-energized, the die closing cylinder is in the pressure holding state, and all hydraulic pumps are unloaded.

(6) Squeeze in cylinder fast forward

At the end of the pressure holding process, the control system sends out the signal, the electromagnet YA2, YA3, YA4, YA5, YA6, YA7, YA8 gets electricity, and the hydraulic oil discharged from the five pumps passes through the one-way valve $1,3,5,7,9$, respectively, through the valve 12 to enter the rear cavity of the cylinder, and the hydraulic oil of the front cavity also enters the rear cavity through valve 13 to realize differential fast advance.

(7) Squeeze into the cylinder

When the extrusion head on the plug rod of the extruded cylinder touches the fast turn and slow limit switch, the electromagnet YA2, YA3, YA5, YA7 gets electricity, and three pumps pass through the one-way valve 1, 3 and 5 respectively, passing through the valve 12 to supply oil to the rear cavity of the extruding cylinder. Squeeze into the cylinder and begin to squeeze the workpiece.

(8) Squeeze into cylinder to hold pressure

After the extruding process of the extrusion head of the extrusion cylinder, the pressure of the system increases, and when the pressure relay SP1 is adjusted, the pressure relay sends out a signal, all electromagnets lose power, all hydraulic pumps are unloaded, and the extrusion cylinder is in a pressure-holding state. The holding time is set by the text display.

(9) Pressure relief, delay

The pressure relief of the right cavity filled with high pressure oil should be carried out before pressing into the cylinder and pressing into the cylinder. This can prevent the phenomenon of gunning and avoid the vibration of the system. After the pressure holding, the signal will be sent out according to the time set by PLC, the electromagnet YA1, YA9 get electricity, squeeze into the cylinder right cavity high pressure relief and delay. The set pressure of the low pressure relief valve at YA1 is $2.5 \mathrm{Mpa}$.

(10) Quick release of the extrusion cylinder

After the workpiece is extruded and molded, the quick-release button is pressed, the electromagnet YA3, YA5, YA8 and YA9 are electrically connected, the valve 13 is opened, the valve 12 is closed, the pumps 3 and 5 are operated, and the hydraulic oil enters the front cavity of the extrusion cylinder and is extruded into the cylinder for quick return.

(11) Pressure relief and delay of mold clamping cylinder

After the pressure holding delay of the die cylinder is finished, the signal is sent by PLC, the electromagnet YA1,
YA12 is electrified, and the high pressure oil unloading delay of the upper cavity of the die cylinder is obtained. The low pressure relief valve at YA1 sets the pressure to $2.5 \mathrm{MPa}$.

(12) Rapid return of die-closing cylinder

After unloading the pressure of the upper cavity of the die-closing cylinder, the PLC control makes the electromagnet YA3, YA11, YA12 get electricity, the plunger pump 2 works, the lower cavity of the pressure oil-closing cylinder, and the upper cavity of the die-closing cylinder are returned to the fuel tank through the one-way valve 21 . The fast return of the die-closing cylinder is realized. The return pressure is adjusted by the pressure regulating valve X11 [4].

At this point, complete a work cycle.

\section{Causes of Vibration}

Figure 1 is a schematic diagram of the hydraulic system of the pipe-end thickener. After careful observation, it is found that the first severe vibration of the system occurs in the extruding cylinder, the unloading stage after the end of the pressure holding, and the second severe vibration of the system occurs in the relief return stage after the closing cylinder is kept in hold. The second vibration is more intense than the first [5]. This shows that the vibration of the system is caused by shock in the process of pressure relief. Since the pressure relief time is too short at the previous time, the pressure relief is not sufficient, resulting in system vibration superposition, and even causing the pipeline to break. By re-adjusting the unloading time, the vibration superposition is avoided and the vibration intensity is reduced, but the vibration of the system is still obvious [6].

First of all, the pressure relief process of the die-closing cylinder is analyzed. Figure 2 is a schematic diagram of the closing cylinder, where a large amount of energy generated by oil compression and deformation energy from the cylinder block and pipeline are accumulated in the upper cavity before the closing cylinder is unloaded. The volume of hydraulic oil in the upper cavity of the closing cylinder is [7]:

$$
V_{1}=\frac{\pi}{4} D_{1}^{2} L_{1}
$$

in the formula: D1-Cylinder diameter $\mathrm{m}$

L1-Total piston stroke $\mathrm{m}$

$$
V_{2}=\frac{\pi}{4} d_{1}^{2} l_{1}
$$

in the formula:

d 1-Tubing inner diameter $\mathrm{m}$

11 - Length of hydraulic pipes connected to the upper cavity of the closing cylinder $\mathrm{m}$. 


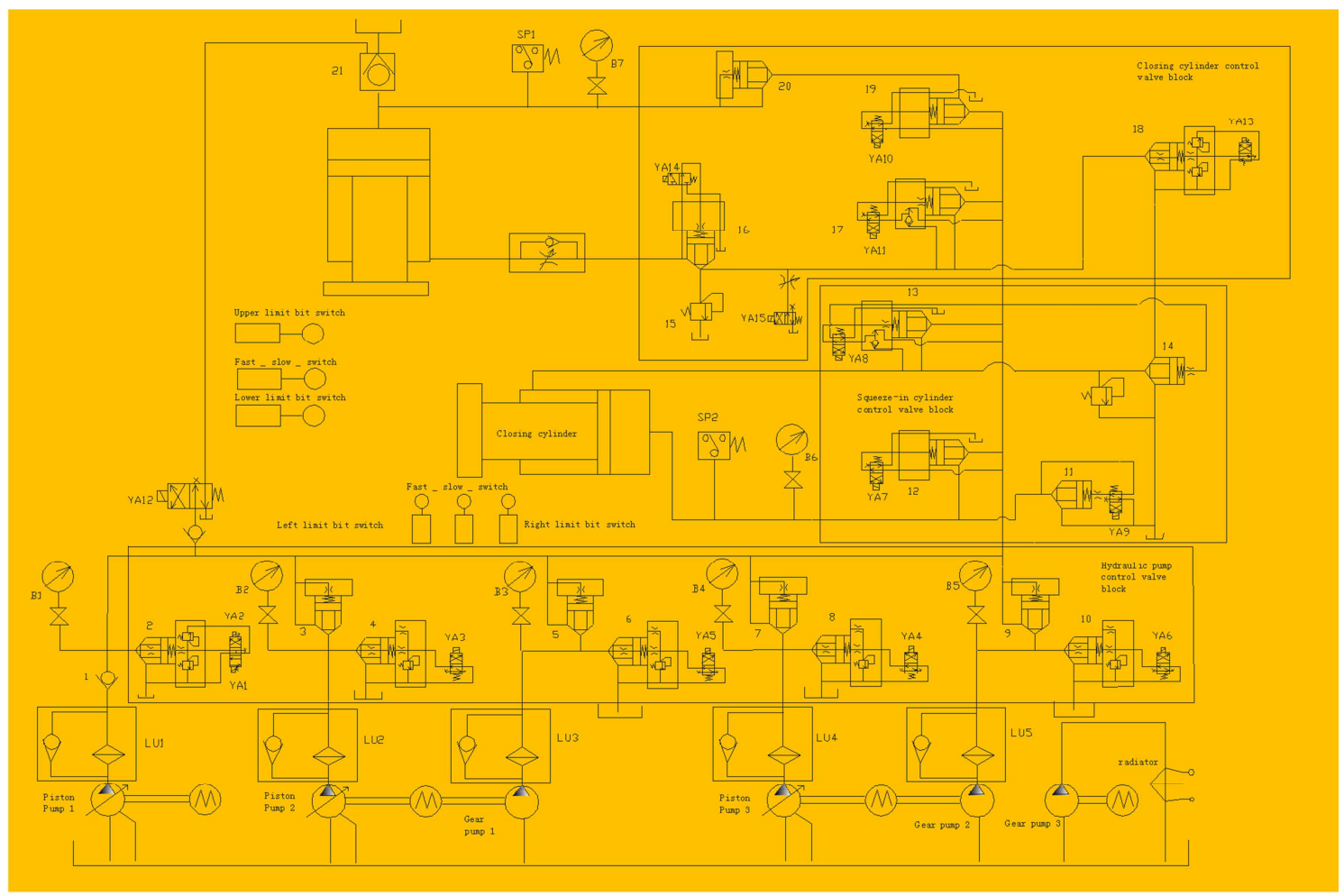

Figure 1. Hydraulic system of pipe end thickener.

Before unloading, the volume of hydraulic oil in the upper cavity of the closing cylinder and its connected tubing is as follows [8]:

$$
\Delta V_{1}=\left(V_{1}+V_{2}\right)\left(p_{1}-p_{2}\right) / k
$$

in the formula:

p1- Pressure before pressure relief pa

P2 - Pressure after unloading pa

$\mathrm{K}$-Volume modulus of hydraulic oil pa

The energy released during the pressure relief of the upper cavity of the mold clamping cylinder is approximately as follows [9]:

$$
\Delta E_{1}=\frac{1}{2}\left(p_{1}-p_{2}\right) \Delta V_{1}
$$

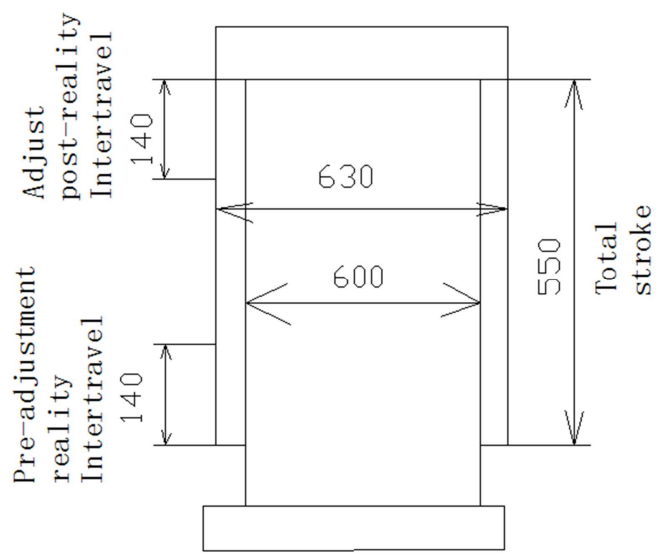

Figure 2. The cylinder for clamping.

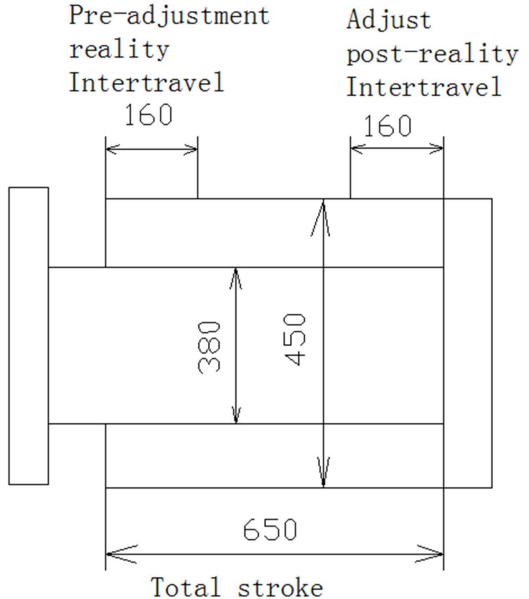

Figure 3. The cylinder for squeeze.

Known: $\mathrm{D} 1=0.63 \mathrm{~m}, \mathrm{~L} 1=0.55, \mathrm{~d} 1=0.063 \mathrm{~m}, 11=10 \mathrm{~m}$, $\mathrm{p} 1=25 \times 106 \mathrm{pa}, \mathrm{p} 2 \approx 0, \mathrm{k}=0.7 \times 109 \mathrm{pa}$. Considering the presence of gas in hydraulic oil, the actual value of calculation is much less than the bulk modulus of pure hydraulic oil. $(1.4 \sim 2) \times 109$ pa. The energy released by substituting the above known data into the (4) form to release pressure from the upper cavity of the closing cylinder is as follows:

$$
\Delta E_{1}=89.3 \times 10^{3} \mathrm{~J}
$$

For the squeeze-in cylinder, the analysis process is similar to the above, as shown in figure 3, Known: Inner diameter of extruded cylinder D2 $=0.45 \mathrm{~m}$, Total stroke of squeeze-in cylinder $\mathrm{L} 2=0.65 \mathrm{~m}$, Inner diameter of connecting extruded 
cylinder line $\mathrm{d} 2=0.063 \mathrm{~m}$, Connecting the length of the extruded cylinder line $11 \mathrm{~m}$ to $8 \mathrm{~m}, 11=8 \mathrm{~m}$. Pressure of extruding cylinder without rod cavity before pressure relief $\mathrm{p} 1$ $=25 \times 106 \mathrm{pa}$, Pressure of extruding cylinder without rod cavity after pressure relief $\mathrm{p} 2 \approx 0, \mathrm{k}=0.7 \times 109 \mathrm{pa}$, The conditional compressibility of the hydraulic oil extruded into the cylinder rod-less cavity and in the pipeline before pressure relief is as follows:

$$
\Delta V_{2}=\frac{\pi}{4}\left(D_{2}^{2} L_{2}+d_{2}^{2} l_{2}\right)\left(p_{1}-p_{2}\right) / k=4.58 \times 10^{-3} m^{3}
$$

The energy released when extruding the cylinder is as follows [10]:

$$
\Delta E_{2}=\frac{1}{2}\left(p_{1}-p_{2}\right) \Delta V_{2}
$$

Calculated:

$$
\Delta E_{2}=57.25 \times 10^{3} \mathrm{~J}
$$

The release of such a large amount of energy in a very short period of time is bound to cause severe vibration and even rupture of the pipeline.

\section{Responses Taken}

In order to avoid the phenomenon of pressure relief shock, the discharge rate should be reduced and the pressure in the process of pressure relief should be reduced monotonously. The average flow rate during decompression is [11]:

$$
Q=\Delta V / t
$$

in the formula:

t-unloading time $s$

As can be seen from formula (8), the pressure relief flow can be reduced by reducing the compression volume $\Delta V$. And prolonging the unloading time $\mathrm{t}$ was obtained. In the pre-adjustment, the system pressure release time has been extended, the vibration intensity has been reduced, and the problem of pipeline rupture has been solved. However, the problem of large vibration of the system has not been solved completely. If the pressure release time is extended, it will reduce the production efficiency and affect the production of enterprises.

Another way to do this is to reduce the volume of compression $\Delta V$. In general, the pressure of the system and the dimensions of the hydraulic cylinder have been determined, and the volume compression of the hydraulic oil $\Delta V$ has been determined. It can't be changed. However, the analysis of the working situation of the two hydraulic cylinders in this system shows that the actual stroke of the hydraulic cylinder can be adjusted in different sections of the total stroke, and the volume of the unloading chamber can be changed, thus the compression volume $\Delta V$ can be changed [12].

$$
V_{1}^{\prime}=\frac{\pi}{4} D_{1}^{2} L_{1}^{\prime}
$$

in the formula $L_{1}^{\prime}$--The actual stroke of the hydraulic cylinder.

Then the compression amount of the hydraulic oil volume in the upper cavity of the die cylinder and its connected tubing becomes:

$$
\Delta V_{1}^{\prime}=\left(V_{1}^{\prime}+V_{2}\right)\left(p_{1}-p_{2}\right) / k
$$

The energy released by the upper cavity of the die cylinder is approximately as follows [13]:

$$
\Delta E_{1}^{\prime}=\frac{1}{2}\left(p_{1}-p_{2}\right) \Delta V_{1}^{\prime}
$$

It is calculated that the energy released from the pressure relief of the upper cavity of the die cylinder becomes:

$$
\Delta E_{1}^{\prime}=31.3 \times 10^{3} \mathrm{~J}
$$

Squeeze into the cylinder has the same problem, make the same adjustment, as shown in figure 3 . The actual stroke of the extruded cylinder is $0.16 \mathrm{~m}$, and the energy released by the extruded cylinder is as follows:

$$
\Delta E_{2}^{\prime}=22.5 \times 10^{3} J
$$

\section{Concluding Remarks}

Through the above adjustment, the energy released by the pressure relief of the die cylinder is reduced to the original. The energy released by the extrusion cylinder is reduced to the original. In this way, not only the energy released during pressure relief is greatly reduced, but also the pressure relief time is greatly shortened [14]. The vibration of the system is greatly reduced, the equipment can run smoothly, the production efficiency is improved, and the power consumption is also reduced [15].

Before adjustment, the piston of the die-closing cylinder works in the lower part of the cylinder block, and the hydraulic oil of the cavity of the die-closing cylinder can not be thoroughly filtered and updated for a long time. The pollutants in the hydraulic oil are precipitated at the upper end of the piston, which aggravates the friction between the piston and the cylinder block, destroys the seal between the cylinder and piston, and increases the internal leakage. After adjustment, the piston works in the upper part of the cylinder block, and the hydraulic oil can be completely recycled and updated, which overcome the problems existing before the adjustment.

\section{References}

[1] Liu Shimei, Tan Jianping, Chen Hui, $300 \mathrm{MN}$ die forging hydraulic press hydraulic pressure system fault simulation study [J]. Forging Technology, 2011, 36 (2) 59/63. 
[2] Yang Guichuan, Yu Jiang, Chen Wen, and so on. Study on the working pressure and working cylinder arrangement of large-scale die-forging hydraulic press $[\mathrm{J}]$. Forging Technology, 2011, 36 (3) 77-79.

[3] Yang Guchuan, Yu Jiang, Chen Wen, et al. Study on frame structure of large die forging hydraulic press [J]. Forging Technology, 2010, 35 (3) 109/112.

[4] He Yanzhong, Chen Wenjia, Huang Jianmin, et al. Design of a new type of travel adjustment mechanism for press [J]. Forging Technology, 2010, 35 (3) 118/121.

[5] Yan Qingsong, Zhang tie, Tong Lei. QA35Y-30 hydraulic combined punching and shearing machine body improvement [J]. Forging Technology, 2010, 35 (4) 72/76.

[6] Han Yukun, Zhang Yunzhen, Wang Lixin, et al. Research on impact dynamic characteristics of $20 \mathrm{MN}$ fast forging hydraulic press [J]. Forging Technology, 2010, 35 (4) 95/98.

[7] Duan Jun, Huang Minghui, Zhanlihua, etc. Research on synchronous balancing system of hydraulic press using PLC and PID [J]. Forging Technology, 2010, 35 (1) 77/80.

[8] Ruan Wei Ping, he Shi Zhen, Wang Enfu. Design analysis and application of hydraulic overload protection device for press $[\mathrm{J}]$. Forging Technology, 2010, 35 (1) 81-83.
[9] Yao Chengyu, Zhao Jingyi, Yang Chenggang. Trouble Analysis and treatment of hydraulic Pneumatic system [M]. Beijing: chemical Industry Press 2009. 12.

[10] Fang Qing. Analysis of pressure-relief performance of CF-type liquid-filling valve [J]. Machine Tools \& Hydraulic, 1998. 2.

[11] Zhang Dapeng, Wu patriotic, du Chunyan, et al. Development of control system for $63 \mathrm{MN}$ forging hydraulic press [J]. Forging Technology, 2009, 34 (6) 109-111.

[12] Li Xinde. Hydraulic system fault diagnosis and maintenance technical manual. Beijing: China Electric Power Publishing House, 2009. 8.

[13] Shen Deliang. Causes and Countermeasures of Pipe burst in Marine hydraulic Piping. [J] Shipbuilding Technology, 1994. 10.

[14] Wisdom. Hydraulic system principle and common fault analysis of high pressure and large flow hydraulic press [J]. Machine tools and hydraulic pressure, 2017. 1.

[15] Wu Bingsheng, Mu Yinbing. Vibration diagnosis of hydraulic system of rolling mill [J]. Hydraulic and Pneumatic, 2014 (1): 128-130. 